seroconverted from positive to negative ("Pos to neg") or remained positive ("Stable pos") or for the specified antibody, between 0-12 months.

anti-CCP2=Anti cyclic citrullinated peptide-2; RF=rheumatoid factor, anti-Car$\mathrm{P}=$ anti carbamylated protein antibodies; Anti-cit. pept. Abs=anti citrullinated peptide antibodies; Anti-acetyl. pept. Abs=anti acetylated peptide antibodies (IgG). Conclusions: Autoantibody levels decrease and seroconversion from positive to negative occurs under treatment, but these changes do not translate to apparent clinical long-term benefits with regard to SDFR. This suggests that the disappearance of measurable serological autoimmunity does not lead to eradication of disease.

REFERENCE:

[1] Heimans AR\&T 2016;18:23.

Disclosure of Interest: None declared

DOI: 10.1136/annrheumdis-2018-eular.6356

\section{THU0070 THE TRANSCRIPTION FACTORS IKAROS AND AIOLOS ARE EXPRESSED IN THE SYNOVIAL MEMBRANE OF EARLY RHEUMATOID ARTHRITIS PATIENTS IN ASSOCIATION WITH SYNOVIAL LYMPHOID AGGREGATES}

F. Rivellese ${ }^{1}$, M. Congia ${ }^{2}$, D. Mauro ${ }^{1}$, M. Bombardieri ${ }^{1}$, M. Lewis ${ }^{1}$, C. Pitzalis ${ }^{1}$. ${ }^{1}$ Centre for Experimental Medicine and Rheumatology, William Harvey Research Institute, Barts and the London School of Medicine And Dentistry, London, UK; ${ }^{2}$ Rheumatology Unit, University and AOU of Cagliari, Monserrato, Italy

Background: IKZF1 (Ikaros) and IKZF3 (Aiolos) are transcription factors acting as regulators of the immune system development. Specifically, they are essential for the maturation, differentiation and survival of B cells. Polymorphisms of IKZF1 and 3 have been linked to systemic autoimmunity, and they are being explored as therapeutic targets in Systemic Lupus Erythematosus. However, their involvement in other autoimmune diseases is currently unknown.

Objectives: To evaluate the expression of IKZF1 and 3 in the synovia of patients with early Rheumatoid Arthritis (RA) naïve to treatment, in correlation with the clinical phenotype, including treatment response.

Methods: DMARD-naïve patients with early ( $<12$ months) RA ( $n=41)$ fulfilling the 2010 ACR/EULAR criteria were recruited as part of the Pathobiology of Early Arthritis Cohort at Barts Health NHS Trust. Sections of paraffin embedded synovial tissue obtained by ultrasound-guided synovial biopsy were stained by immunohistochemistry (IHC) for IKZF1 and IKZF3, and a semi-quantitative score (0-3) was used to classify patients (IKZF1 +ve or IKZF3 +ve cells/visual field $<5=0 ; 5-$ $20=1 ; 20-50=2 ;>50=3$ ). Sequential sections were stained by $\mathrm{IHC}$ for immune cells and patients were categorised into 3 synovial pathotypes according to the following criteria: i) Lymphoid (L) presence of grade 2-3 CD20 +aggregates, (CD20 $\geq 2$ ) and/or CD138 >2; ii) Myeloid (M) CD68SL $\geq 2$, CD20 $\leq 1$ and/or CD3 $\geq 1$, CD138 $\leq 2$ and iii) Fibroid (F) CD68SL $<2$ and CD3, CD20, CD138 $<1$.

Results: Ikaros and Aiolos were expressed in the synovia of $43.1 \%$ and $56.7 \%$ of early RA patients, respectively. IKZF1 +ve patients (defined as IKZF1 score $\geq 2$ ) showed a higher prevalence of a lymphoid pathotype (9/9 in IKZF1 +ve vs 4/22 in IKZF1-ve, $\mathrm{p}<0.001)$ and a higher prevalence of ACPA (9/9 vs 13/22, $\mathrm{p}=0.04)$ and RF $(9 / 9$ vs $12 / 22, p=0.02)$. IKZF3 + ve patients showed a similar association with local and systemic inflammation and autoantibody positivity. As shown in table 1, ikaros and aiolos synovial scores were significantly correlated with synovial cell infiltrate and systemic inflammation. Remarkably, ikaros showed a significant correlation with the baseline Sharp score. Accordingly, patients with high expression of Ikaros (sq score $\geq 2$ ) had a significantly higher Sharp score (mean \pm SD 4.75 \pm 3.3 vs $1.33 \pm 2.54, p=0.008$ )

Conclusions: Here, we show the expression of the transcription factors lkaros and Aiolos in the synovia of early RA patients in correlation with lymphoid aggregates and systemic inflammation, and, for Ikaros, with baseline radiographic erosions. While additional analyses are needed in order to confirm the expression and function of ikaros and aiolos by synovial immune cells, our preliminary work suggest that they might be relevant in the pathogenesis of RA and therefore be considered as therapeutic targets in a subset of patients.

Disclosure of Interest: None declared

DOI: 10.1136/annrheumdis-2018-eular.6442

\section{THU0071 \\ MUSCLE LIPOTOXICITY ON SARCOPENIA DEVELOPMENT IN A MODEL OF COLLAGEN-INDUCED ARTHRITIS}

G. Vial ${ }^{1}$, A. Pinel ${ }^{2}$, D. Bechet ${ }^{2}$, F. Wauquier ${ }^{2}$, Y. Wittrant ${ }^{2}$, C. Chevenet ${ }^{3}$, M. Soubrier ${ }^{1,2}$, A. Tournadre ${ }^{1,2}$, F. Capel ${ }^{2}$. ${ }^{1}$ Rheumatology, CHU Gabriel Montpied; ${ }^{2}$ INRA; ${ }^{3}$ Anatomical Pathology, CHU Gabriel Montpied, Clermont Ferrand, France

Background: Alterations of body composition in Rheumatoid arthritis (RA) may contribute to the development of cardiometabolic disorders. RA patients have a decrease in muscle mass with a preserved or increased fat mass, notably accumulation of ectopic fat in the muscles, witch define the sarcopenic obesity. The mechanisms leading to this sarcopenic obesity phenotype remain poorly understood. Accumulation of intramuscular lipids and the formation of lipotoxic compounds may affect intracellular signalling pathways and energy production, alter protein synthesis and thus promote sarcopenia.

Objectives: Our objective was to determine if muscular lipotoxicity promotes the development of sarcopenia in joint inflammatory condition, using rats with collagen-induced arthritis.

Methods: Male Sprague Dawley rats were divided into a control group (CO, $n=12)$ and a collagen-induced arthritis group (CIA, $n=11)$. After 5 weeks, hind leg muscles and epididymal adipose tissue were removed. Tissues were weighed at sacrifice and stored for histological analyses and evaluation of mitochondrial func tion, lipotoxicity, protein and gene expression levels. Statistical analyses were performed with t-test.

Results: Animals, adipose tissue and muscle weights tended to be lower in the CIA group. Only EDL muscle weight was significantly lower in the CIA group $(p=0.05)$. Muscle histological analyses showed larger CSA (cross sectional area) in $\mathrm{CO}$ group. In the CIA group we observed a nonhomogeneous distribution of muscle fibre CSA with a predominance of small fibres (figure 1). Mean perimeter and mean diameter were also significantly decrease in CIA group but the shape of fibres remained similar between groups. Furthermore, there was an increased expression of MAFBx (a marker of catabolism) mRNA $(40 \%, p=0.04)$ in the CIA group, while MyoD (a myogenesis marker) mRNA was decreased by $18 \%$ $(p=0.01)$, indicating a catabolic state. Lipid content analysis showed an accumulation of intramuscular TAG ( $\times 1.5, \mathrm{p}=0.05)$, as well as an increased expression of cellular fatty acid transporter FATP1 (about 35\%, $\mathrm{p}=0.01$ ) and mitochondrial fatty acid transporter CPT1b (about 27\%, $\mathrm{p}=0.02$ ). Mitochondrial DNA copy number was decreased by $27 \%$ in CIA rats $(p=0.01)$ and complex IV activity of mitochondrial respiratory chain also tended to be reduced in CIA group $(-20 \%, p=0.18)$.

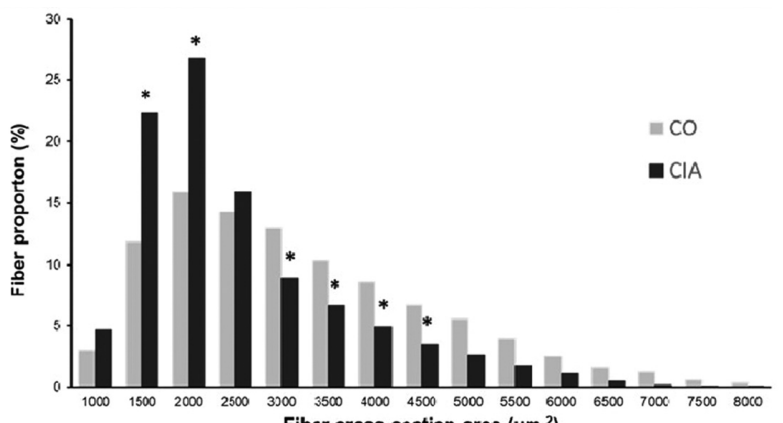

Abstract THU0071 - Figure 1. Fibre cross sectional area distribution

Conclusions: Collagen-induced arthritis induced fibres alterations in skeletal muscle. The association of increased muscle protein catabolism, mitochondrial dysfunction and fatty acid accumulation in skeletal muscle of animals with arthritis supports the hypothesis that lipotoxicity is involved in sarcopenia development during joint inflammation.

Disclosure of Interest: None declared

DOI: 10.1136/annrheumdis-2018-eular.5978

Abstract THU0070 - Table 1. Spearman correlation coefficients

\begin{tabular}{lcccccccrrr}
\hline & Krenn & CD3 & CD20 & CD68L & CD68SL & CD138 & ESR & CRP & DAS28 & Sharp \\
\hline IKZF1 & $0.659^{* *}$ & $0.712^{* *}$ & $0.816^{* *}$ & $0.525^{* *}$ & $0.432^{*}$ & $0.851^{* *}$ & $0.553^{* *}$ & $0.491^{* *}$ & 0.333 & $0.410^{*}$ \\
IKZF3 & $0.658^{* *}$ & $0.652^{* *}$ & $0.809^{* *}$ & $0.581^{* *}$ & $0.550^{* *}$ & $0.812^{* *}$ & $0.487^{*}$ & $0.474^{*}$ & 0.296 & 0.313 \\
\hline
\end{tabular}

${ }^{*} p<0.05 ;{ }^{* *} p<0.01$ 